REVIEW SERIES

\title{
Sleep - 4: Sleepiness, cognitive function, and quality of life in obstructive sleep apnoea/hypopnoea syndrome
}

\author{
H M Engleman, N J Douglas
}

Thorax 2004;59:618-622. doi: 10.1136/thx.2003.015867

Sleepiness, cognitive performance, and quality of life are overlapping aspects of daytime function that may be affected in patients with obstructive sleep apnoea/ hypopnoea syndrome. The evidence is compatible with hypotheses that these deficits are reversible with treatment, particularly for patients with severe disease.

$\mathrm{T}$ he daytime consequences of the obstructive sleep apnoea/hypopnoea syndrome (OSAHS) are usually more important to the patient than the nocturnal events on which clinicians tend to focus. Patients may be personally unaware of their snoring and breathing pauses during sleep, but acutely aware of its consequences in the form of daytime sleepiness, impaired work performance, irritability, marital disharmony, and reduced participation in and enjoyment of everyday activities. This selective review compares and contrasts functional consequences of OSAHS in the form of impairments to daytime alertness, ${ }^{1-28}$ cognitive performance, ${ }^{12} 17222325-34$ and quality of life. ${ }^{12} 1722-2835-44$

Sleepiness, cognitive performance, and quality of life are interrelated aspects of daytime function for patients with OSAHS, each impinging on the others. However, specific tests have been developed to assess function within these separate areas. There is evidence of impairment in each of these areas but, to allow comparisons, it is convenient to convert scores to a common metric - the effect size. ${ }^{45}$ Effect sizes are calculated by dividing the mean difference in score by its standard deviation (SD), thus estimating how many standard deviations the impairment or treatment has moved in the population under study. Case-control differences express impairment effect sizes, and active-placebo treatment differences therapeutic effect sizes. Effect sizes of $0.25 \mathrm{SD}$ or less are considered clinically small differences, those up to $0.5 \mathrm{SD}$ moderate, and those of $0.75 \mathrm{SD}$ or more of clinically large magnitude. ${ }^{45}$

See end of article for authors' affiliations

......................

Correspondence to: Dr H M Engleman, Edinburgh Sleep Centre, Royal Infirmary of Edinburgh, Lauriston Place, Edinburgh EH3 9YW, UK; h.engleman@ ed.ac.uk

\section{SLEEPINESS}

Excessive daytime sleepiness is the most common daytime symptom in OSAHS $^{1}$ and frequently forms a selection criterion for defining patient samples for research and clinical practice. Sleepiness has been assessed using a variety of subjective and objective means, and in clinical studies $^{1-28}$ these tend to indicate at least moderate impairments both to subjective and objective
Subjective ratings of sleepiness include the Profile of Mood States (POMS), ${ }^{23}$ Stanford sleepiness scale $^{310}$ and Epworth sleepiness scale. $^{4-822-29}$ These first two instruments are "state" ratings of sleepiness reflecting mood at the time of rating, while the Epworth scale seeks ratings of recent sleepiness over the previous month.

State sleepiness ratings by the POMS indicate substantially reduced vigour $^{23}$ and increased fatigue $^{3}$ scores in small samples of patients with OSAHS, with mean values deviating from those of normal controls ${ }^{2}$ by 1.5-2 SD. For vigour, controls have a mean (SD) of 23 (4) points compared with 14 or 15 points in patients with OSAHS, ${ }^{23}$ and for fatigue patients have a mean of 8 or 10 points, respectively, against normal mean (SD) values of 4 (4) points.

The Stanford sleepiness scale shows more subtle differences in clinical studies, ${ }^{3}{ }^{10}$ with scores not always significantly different from normal controls. ${ }^{10}$ The Epworth scale ${ }^{4-822-29}$ is now the most commonly used rating of sleepiness in sleep disorders research. It asks subjects to rate their likelihood of dozing in eight everyday situations associated with greater to lesser sleepiness from "watching TV" to "driving a car". It may provide less bias than other selfrating scales by asking about the last month's sleepiness rather than about the instantaneous mood state, as rated by state measures. This could improve the reliability when providing self-ratings in a stimulating laboratory or clinic environment where sleepiness might be reduced by situational factors. The focus of the Epworth scale on a behaviour (dozing) rather than an internal mood may be an advantage in circumstances where patients have become so accustomed to persistent sleepiness that they lose their frame of reference and fail to recognise their sleepiness as pathological. The Epworth scale yields typical mean scores of $16,{ }^{6} 15,{ }^{7}$ and $14^{4}$ out of 24 in OSAHS patients with a typical SD of $4 .^{5}$ Thus, patients with classical OSAHS score 2 or more SD above the average 5 in healthy working normal subjects, ${ }^{5}$ representing a very large impairment effect size. alertness in typical patients with OSAHS.
Abbreviations: $\mathrm{AHI}$, apnoea + hypopnoea index; CPAP, continuous positive airway pressure; FOSQ, functional outcomes of sleepiness questionnaire; HADS, hospital anxiety and depression scale; MSLT, multiple sleep latency test; MWT, maintenance of wakefulness test; NHP, Nottingham health profile; OSAHS, obstructive sleep apnoea/hypopnoea syndrome; OSLER, Oxford sleep resistance test; POMS, profile of mood states; SAQLI, sleep apnoea quality of life index 
Objective tests of daytime sleepiness include the multiple sleep latency test (MSLT), ${ }^{10-17} 222527$ the maintenance of wakefulness test (MWT), ${ }^{14} 202126$ and the OSLER (Oxford SLEep Resistance) test. $^{18} 1923$ The MSLT is a polysomnographic test of sleep tendency requiring subjects to try to fall asleep during four or five daytime nap opportunities lasting 20 minutes or less. The latency from lights out to sleep onset is the principal outcome measure. Patients with severe OSAHS often have mean sleep onset latencies in the pathological range of 5 minutes or less, ${ }^{10}{ }^{11}$ some 2 SD below normal mean (SD) values of 12 (4). In one sample of UK patients with classical OSAHS, ${ }^{22}$ placebo values for MSLT sleep onset latency averaged 7 minutes, providing an impairment effect size of 1.3 SD. Other studies providing baseline MSLT values from later and probably less severe OSAHS patients confirm the impression of at least mild to moderate sleepiness, ${ }^{3}{ }^{12-17} 22$ demonstrating sleep onset latencies consistently below the normal threshold of 10 minutes. In these patients, impairment effect sizes average approximately $1 \mathrm{SD}$.

The MWT is also polysomnographic but uses an inverse paradigm requiring the subject to stay awake as long as possible under soporific conditions. ${ }^{14202126}$ One case-control study using an MWT-like paradigm has provided evidence of large magnitude decrements in sleep onset latency in OSAHS patients averaging 16 minutes compared with healthy controls with mean (SD) latencies of 27 (8) minutes. ${ }^{20}$ Here, the impairment effect size is estimated at $1 \mathrm{SD}$.

A recently developed tool, the Oxford sleep resistance test (OSLER), employs an MWT-like environment and instruction to stay awake but is based on a behavioural and not a polysomnographic signal-namely, lapses in button pressing responses to a repeated visual stimulus. ${ }^{18}$ The OSLER discriminates normal subjects (mean latency to lapsing 40 minutes) from OSAHS patients (10 minutes), has good concurrent validity with the MWT, and "lapsing" on the OSLER has been validated against polysomnographically defined microsleeps. ${ }^{19}$ The OSLER may thus offer a low technology alternative to the polysomnographic tests.

Estimates of average impairment effect sizes using these subjective and objective tools tend to indicate very large increments in sleepiness in OSAHS patients compared with controls (fig 1). In objective tests of sleepiness such as the MSLT and MWT, impairment effect sizes approximate 1.5$2 \mathrm{SD}$, and for subjective outcomes such as the Epworth scale these may be as large as $2.5 \mathrm{SD}$.

An association between severity of OSAHS and subsequent sleepiness is somewhat supported by clinical ${ }^{8-10} 151621$ and epidemiological studies ${ }^{9}$ employing correlative techniques. In a large community sample ${ }^{9}$ subjective hypersomnolence rose in a dose-responsive manner with the level of sleep disordered breathing. In studies of OSAHS patients, some drawing upon substantial sample sizes, ${ }^{8} 151621$ weak to

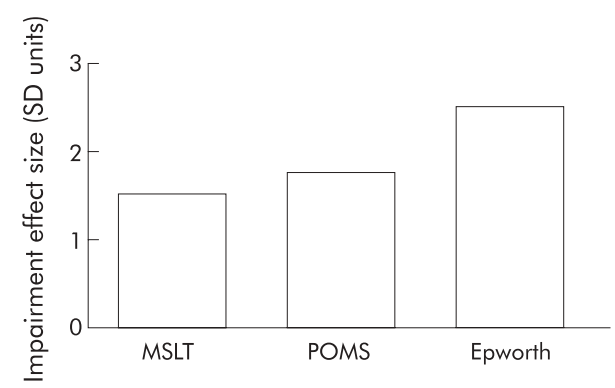

Figure 1 Impairment effect sizes from tests of sleepiness in moderate and severe OSAHS. MSLT = multiple sleep latency test; POMS = profile of mood states. moderate relationships $(r=0.3-0.5)$ between the apnoeahypopnoea index (AHI) and subjective ${ }^{8}$ and objective daytime sleepiness ${ }^{10} 1521$ have been demonstrated. Other stu$\operatorname{dies}^{812}{ }^{16}$ have found no significant correlation between AHI and MSLT scores and, in those with smaller sample size, ${ }^{8}{ }^{12}$ this may reflect both the weak relationship and limited statistical power.

Thus, case-control studies suggest that impairments of daytime sleepiness-whether self-rated or measured by objective instruments-are large in patients with OSAHS. Subjective assessments probably show larger effects than objective measures. Correlational analyses suggest that sleepiness may be at least partially determined by the severity of nocturnal events in patients.

\section{COGNITIVE PERFORMANCE}

One of the potential consequences either of nocturnal hypoxaemia or severe sleepiness is the impairment to cognitive performance both reported by ${ }^{15-37}$ and measured in $^{11} 121722$ 25-33 patients with OSAHS. Cognitive impairments may be an important co-factor in the increased risk of road traffic accidents in OSAHS, a subject to be covered in another review in this series.

In older case series of patients with severe OSAHS, over three quarters reported difficulties at work and reduction in work capacity and efficiency. ${ }^{35}$ Even in more recent samples the prevalence of such perceived problems remains high, with approximately two thirds of new patients still reporting difficulties in work efficiency and performing new tasks. ${ }^{36}$ In a disease specific symptom inventory, ${ }^{43}$ two thirds experienced memory disturbance and three quarters problems with concentration.

Objective assessments of cognitive performance in OSAHS have been conducted using a variety of neuropsychological tests, usually conducted with pen and paper or by computer. ${ }^{46}$ Cognitive scores have been collected in community based epidemiological studies ${ }^{30} 31$ and clinical case-control studies (table 1), ${ }^{11}{ }^{32} 33$ which can then be ranked in order of mean severity of sleep disordered breathing. Outcomes from the tests of neuropsychological performance, although intercorrelated, may be broadly divided into the functional areas of attention, memory and learning, and executive performance (planning skill and problem solving). A plot of mean impairment effect sizes ${ }^{46}$ within these three cognitive areas shows very small impairments in community recruited subjects with low levels of sleep disordered breathing, many not statistically different from those of normal subjects at 0.3 SD or less. However, moderate or large impairment effect sizes are obtained from the clinical studies of patients diagnosed with OSAHS which broadly increase with mean AHI. Mean impairment effect sizes from this plot, weighted for subject sample size, are estimated at 1, 0.5, and 0.9 SD, respectively, for attention, memory, and executive performance at a grand mean AHI for all studies of 30 events/hour.

This analysis suggests that OSAHS of moderate severity may be associated with moderate to large impairments on

Table 1 Community and clinical studies of cognitive performance

\begin{tabular}{lrl}
\hline & No & Mean AHI \\
\hline Redline $^{30}$ & 32 & 17 \\
Kim $^{31}$ & 199 & 19 \\
Bedard" $_{\text {Naegel }^{33}}$ & 10 & 21 \\
Greenberg $^{32}$ & 17 & 41 \\
Bedard'1 $^{\text {11 }}$ & 14 & 48 \\
\hline
\end{tabular}


tests of cognitive performance, but that significant impairments may not be evident at low levels of sleep disordered breathing and symptomatology.

Cognitive dysfunction associated with OSAHS appears to be smaller than the increases in sleepiness, where impairment effect sizes typically exceed I SD. This is compatible with a pathophysiological mechanism where at least some cognitive decrements are a secondary consequence of sleepiness, and thus variably expressed in the symptomatology of patients with OSAHS. This hypothesis remains open to further study but is also supported by experimental evidence $^{34}$ of sleepiness and cognitive outcomes after sleep fragmentation in normal subjects. Here, one night of regular, brief auditory sleep disruption produced moderate to large impairments in sleepiness outcomes $(0.75-1$ SD) but small to moderate decrements in attention biased cognitive scores (fig 2).

\section{QUALITY OF LIFE}

Patients with OSAHS have significantly impaired quality of life $^{41-44}$ and social functioning ${ }^{35}{ }^{37}$ and a high prevalence of minor psychiatric morbidity. ${ }^{12} 1722$ The mechanisms producing these have not been investigated in detail, but it is possible that they are related sequelae of pervasive sleepiness, reducing mood, motivation and performance with impact on wide ranging psychological and social function.

Early studies in patients with severe OSAHS suggested that more than two thirds had problems in social and interpersonal function, ${ }^{35}$ especially in the areas of work performance and marital and family relationships. Such broad aspects of life quality were also assessed in a large epidemiological survey of overweight subjects. This showed that those with symptoms of OSAHS had poorer perceived health than asymptomatic subjects, lower economic income, and increased odds of having had psychiatric care, multiple divorce, and impaired work performance. ${ }^{37}$

One of the chief consequences of impaired quality of life may be minor psychiatric illness such as anxiety and depressive disorders. Screening instruments such as the Zung depression scale, ${ }^{39}$ the Hospital Anxiety and Depression scale (HADS), ${ }^{12} 17222526$ and the General Health Questionnaire- $28^{22}$ identify significant psychiatric symptoms in approximately one third to one half of patients with OSAHS. ${ }^{12} 172239$

Recent research in quality of life, drawing on larger samples, has used generic quality of life scales to assess the functional limitations imposed by OSAHS. Instruments have included the Nottingham Health Profile (NHP) 22243840 and the medical outcomes study short form-36 questionnaire (SF-36). ${ }^{2326274142}$ The NHP, a questionnaire of perceived health status containing subscales for energy, pain, emotional reactions, sleep quality, social isolation, and physical

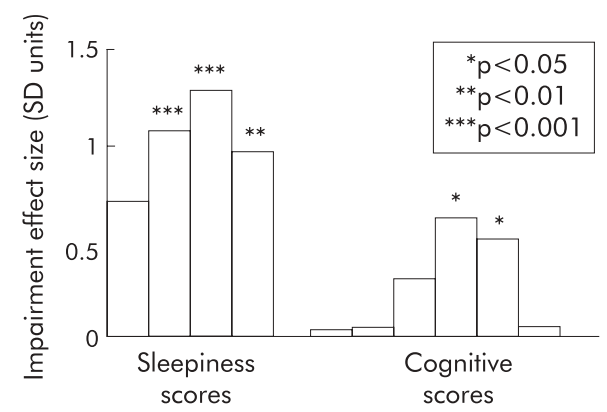

Figure 2 Sleepiness and cognitive performance impairment effect sizes following sleep fragmentation in normal subjects. Data from Martin et al. ${ }^{34}$ mobility, revealed significant impairments in all dimensions except emotional function in 103 patients with OSAHS compared with a similarly aged control group of nonsnorers. ${ }^{40}$ The SF-36, which includes subscales for physical function, role-physical, role-emotional, pain, general health, social function, vitality and mental health, has also indicated considerable impairments in UK samples of patients with OSAHS. ${ }^{23} 264142$ The most severely affected subscore in these clinical studies is that for vitality, in keeping with a clinical picture of sleepiness as a dominant symptom in OSAHS. However, quality of life scores are also reduced for the other domains of function and health.

In one study using the SF-36, ${ }^{41}$ broadly dose related increases in the number and magnitude of subscale impairments were documented, with the most severe patients by symptomatic and oximetric measures showing impairment effect sizes averaging 1 SD in size in all subscales compared with normative scores. The SF-36 subscores in another group of patients with severe OSAHS showed congruent large and broad ranging impairments effects. ${ }^{42}$ Some $80 \%$ of this patient sample had below average scores in one or both of the SF-36 summary indices-the mental and physical component scores. In patients with milder polysomnographic illness but substantial symptoms, ${ }^{26}$ similar impairments were observed.

Illness-specific scales assessing the impact of OSAHS have also been developed, including the Calgary sleep apnoea quality of life index (SAQLI) $)^{43}$ and the functional outcomes of sleepiness questionnaire (FOSQ). ${ }^{44}$ For the FOSQ subscales, large impairment effect sizes of $\geqslant 1$ SD are found in patients with OSAHS, ${ }^{44}$ as might be expected of an instrument designed to investigate specific symptoms of sleepiness.

Research using generic and illness-specific measures suggests that quality of life in OSAHS is substantially reduced, with limitations occurring both in mental and physical function. The existing literature is compatible with the hypothesis that such functional impairments are partially mediated by symptomatic severity.

\section{REVERSIBILITY WITH TREATMENT}

Controlled clinical trials of treatments for OSAHS ${ }^{22-29}$ provide a tool to examine the reversibility of the daytime impairments associated with the syndrome. Many recent randomised controlled studies have included outcomes from tests of sleepiness, cognitive performance, and quality of life, allowing therapeutic effect sizes to be compared and contrasted in varying patient populations.

In patients with classical OSAHS ${ }^{22-24}$ the therapeutic effect sizes for sleepiness after continuous positive airway pressure (CPAP) are moderate to large. The OSLER sleep onset latency improved by $0.7 \mathrm{SD}$ while subjective Epworth and vitality scores showed very large improvements of 1.5 and 2 SD, respectively. ${ }^{23}$ A similar very large improvement in Epworth score (2 SD) was reported in another randomised controlled trial. ${ }^{24}$ In a slightly milder but still symptomatic group of 48 patients with AHI of $\geqslant 15$ there was an average therapeutic effect from CPAP of 0.5 SD in objective sleepiness by MSLT sleep onset latency and 0.9 SD in Epworth score. ${ }^{22}$ These findings are again compatible with OSAHS having a greater effect on subjective than objective sleepiness.

In classical OSAHS, changes to cognitive performance scores after treatment are smaller in size and sparser in appearance than those for sleepiness. Most outcomes in a computer administered driving based task were significantly improved in one trial of CPAP, ${ }^{29}$ with small to moderate improvements of $0.5 \mathrm{SD}$ or less against the large and very large improvements observed in sleepiness. Another study showed that CPAP produced significant improvements in four of eight attention biased cognitive test outcomes, but 


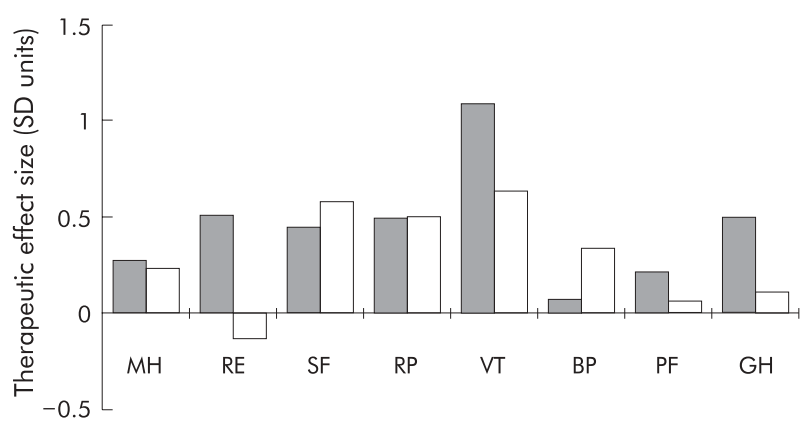

Figure 3 Therapeutic effect sizes for SF-36 subscores. Data for patients with severe (shaded bars) ${ }^{23}$ and mild OSAHS (white bars). ${ }^{26}$

$\mathrm{MH}=$ mental health; $\mathrm{RE}=$ role emotional; $\mathrm{SF}=$ social function; $\mathrm{RP}=$ role physical; $\mathrm{VT}=$ vitality; $\mathrm{BP}=$ bodily pain; $\mathrm{PF}=$ physical function; $\mathrm{GH}=$ general health.

again with small to moderate effect sizes (0.2-0.4 SD), much smaller than those for sleepiness. ${ }^{22}$

Quality of life measures in randomised controlled trials of patients with moderate to severe OSAHS appear to improve with treatment as a function of initial impairment, with the worst affected subscale scores showing the greatest improvements. In the randomised controlled trial conducted by Jenkinson et al ${ }^{23}$ very large improvements were seen in vitality score, with large effect sizes for social function and moderate or small increments for the other subscale scores (fig 3). In another controlled study using the NHP, Ballester et $a^{24}$ found small therapeutic effects for all subscores except the energy scale which showed a large improvement (0.75 SD). Thus, in classical OSAHS the large impairments in sleepiness and energy related quality of life scores appear to show substantial therapeutic improvement and the moderate impairments of cognitive performance show small improvements.

In patients with OSAHS of milder polysomnographic severity significant therapeutic effects in randomised controlled trials ${ }^{25-28}$ are fewer and hard to demonstrate, especially for objective outcomes. Among these studies, therapeutic effect sizes for the Epworth score were $0.0 \mathrm{SD}^{25} 0.7 \mathrm{SD}^{26}$ $0.1 \mathrm{SD}^{27}$ and $0.3 \mathrm{SD}^{28}$ yielding a mean change of $0.3 \mathrm{SD}$. Respective changes in objective sleepiness for these were $0 \mathrm{SD}^{25} 0.2 \mathrm{SD},{ }^{26} 0.2 \mathrm{SD},{ }^{27}$ and $0.0 \mathrm{SD},{ }^{28}$ providing a mean therapeutic effect size of 0.1 SD. Multiple tests were used to assess cognitive performance across these four studies. However, statistically significant improvements in milder patients were found for one of eight tests, ${ }^{25}$ two of seven, ${ }^{26}$ one of five, ${ }^{27}$ and none of six. ${ }^{28}$ Thus, four of 26 cognitive scores were significantly improved-a proportion higher than the one significant test expected by chance alone. Effect sizes in these significant performance scores were 0.3-0.5 SD, in the small to moderate range. For quality of life scores the SF36 has shown significant improvements with moderate effect sizes in five of nine subscores in one study ${ }^{26}$ but none in another. $^{27}$ As in patients with more severe OSAHS, the subscore showing the largest therapeutic effect was that for vitality $(0.7 \mathrm{SD})$. Other quality of life related scores from the FOSQ $^{27}{ }^{28}$ and the NHP ${ }^{25}{ }^{28}$ did not improve in some trials, but HADS depression scores from the other two trials ${ }^{25} 26$ did show moderate enhancements of 0.4 SD with CPAP.

The four studies of mild OSAHS sampled somewhat different populations with mean Epworth scores of 14, 13, 11 , and 12, respectively. ${ }^{25-28}$ Broadly, the size and range of therapeutic improvements increased with higher sleepiness, compatible with sleepiness being a determinant of impairment and thus improvement. Some researchers have noted a comparative absence of initial impairment to sleepiness, cognitive function, and quality of life in those with mild polysomnographic illness and lower symptomatic status in terms of sleepiness. ${ }^{27} 28$

\section{CONCLUSIONS}

Sleepiness, cognitive performance, and quality of life are overlapping aspects of daytime function that may be affected in patients with OSAHS. Varying objective and subjective tests have been developed and used in clinical studies to assess these, making comparisons across measurements and studies problematic. An overview of sleepiness, cognitive function, and quality of life in OSAHS is facilitated by converting varying test scores to standardised effect sizes which estimate impairment and therapeutic effects in SD units. Effect sizes for sleepiness impairment in patient samples with severe OSAHS are large for objective tests $(\sim 1.5 \mathrm{SD})$ and larger still $(\sim 2.5 \mathrm{SD})$ for subjective ratings. Cognitive performance scores seem minimally affected in community samples with mild sleep disordered breathing, but decrements become moderate $(\sim 0.5 \mathrm{SD})$ or large $(>0.7 \mathrm{SD})$ in clinical samples of OSAHS patients. Ratings of quality of life and well being show high prevalences of reduced functioning and psychiatric morbidity in patients and broad ranging impairments across physical and mental function, with the largest effects ( $>1$ SD) for subscores reflecting energy and vitality. The evidence is compatible with hypotheses that these deficits are reversible with treatment, although with larger improvements in subjective than objective tests and a broader range of enhancements for patients with more severe disease.

\section{Authors' affiliations}

H M Engleman, N J Douglas, Edinburgh Sleep Centre, Unit of Respiratory Medicine, University of Edinburgh, UK

\section{REFERENCES}

1 Guilleminault C, van den Hoed J, Mitler MM. Clinical overview of the sleep apnea syndromes. In: Guilleminault C, Dement WC, eds. Sleep apnea syndromes. New York: Alan R Liss, 1978:1-12.

2 Beutler LE, Ware JC, Karacan I, et al. Differentiating psychological characteristics of patients with sleep apneas and narcolepsy. Sleep $1981 ; 4: 39-47$.

3 Kribbs NB, Pack Al, Kline LR, et al. Effects of one night without nasal CPAP treatment on sleep and sleepiness in patients with obstructive sleep apnea. Am Rev Respir Dis 1993; 147:1162-8.

4 Johns MW. Reliability and factor analysis of the Epworth sleepiness scale. Sleep 1992;15:376-81.

5 Johns M, Hocking B. Daytime sleepiness and sleep habits of Australian workers. Sleep 1997;20:844-9.

6 Hardinge FM, Pitson DJ, Stradling JR. Use of the Epworth sleepiness scale to demonstrate response to treatment with nasal continuous positive airway pressure in patients with obstructive sleep apnoea. Respir Med 1995;89:617-20.

7 Engleman HM, Asgari-Jirhandeh N, McLeod AL, et al. Self-reported use of CPAP and benefits of CPAP therapy. Chest 1996;109:1470-6.

8 Johns MW. Daytime sleepiness, snoring and obstructive sleep apnea: the Epworth sleepiness scale. Chest 1993;103:30-6.

9 Young T, Palta M, Dempsey J, et al. The occurrence of sleep-disordered breathing among middle-aged adults. N Eng J Med 1993;328:1230-5.

10 Roth T, Hartse KM, Zorick F, et al. Multiple naps and the evaluation of daytime sleepiness in patients with upper airway sleep apnea. Sleep 1980;3:425-39.

11 Bédard M-A, Montplaisir J, Richer F, et al. Obstructive sleep apnea syndrome: pathogenesis of neuropsychological deficits. J Clin Exp Neuropsychol $1991 ; 13: 950-64$

12 Cheshire K, Engleman H, Deary I, et al. Factors impairing daytime performance in patients with sleep apnea/hypopnea syndrome. Arch Intern Med 1992; 152:538-41.

13 Lamphere J, Roehrs T, Wittig R, et al. Recovery of alertness after CPAP in apnea. Chest 1989;96:1364-7.

14 Sangal RB, Thomas L, Mitler MM. Disorders of excessive sleepiness: treatment improves ability to stay awake but does not reduce sleepiness. Chest 1992;102:699-703.

15 Roehrs T, Zorick F, Wittig R, et al. Predictors of objective level of daytime sleepiness in patients with sleep-related breathing disorders. Chest 1989;95:1202-6.

16 Guilleminault C, Partinen M, Quera-Salva MA, et al. Determinants of daytime sleepiness in obstructive sleep apnea. Chest 1988;94:32-7. 
17 Engleman HM, Cheshire KE, Deary IJ, et al. Daytime sleepiness, psychometric performance and mood after CPAP therapy for the sleep apnoea/hypopnoea syndrome. Thorax 1993;48:911-4.

18 Bennett LS, Stradling JR, Davies RJO. A behavioural test to assess daytime sleepiness in obstructive sleep apnoea. J Sleep Res 1997:6:142-5.

19 Priest B, Brichard C, Aubert G, et al. A validation study of the OSLER test. Am J Respir Crit Care Med 2001;163:1619-25.

20 Sforza E, Krieger J. Daytime sleepiness after long-term continuous positive airway pressure (CPAP) treatment in obstructive sleep apnea syndrome. J Neurol Sci 1997;110:21-6.

21 Poceta JS, Timms RM, Jeong D-U, et al. Maintenance of wakefulness test in obstructive sleep apnea syndrome. Chest 1992;101:893-7.

22 Douglas NJ. Systematic review of the efficacy of CPAP. Thorax 1998:53:414-5.

23 Jenkinson C, Davies RJ, Mullins R, et al. Comparison of therapeutic and subtherapeutic nasal continuous positive airway pressure for obstructive sleep apnoea: a randomised prospective parallel trial. Lancet 1999;353:2100-5.

24 Ballester E, Badia JR, Hernández L, et al. Evidence of the effectiveness of continuous positive airway pressure in the treatment of sleep apnea/ hypopnea syndrome. Am J Respir Crit Care Med 1999;159:495-501.

25 Engleman HM, Martin SE, Deary IJ, et al. Effect of CPAP therapy on daytime function in patients with mild sleep apnoea/hypopnea syndrome. Thorax 1997:52:114-9.

26 Engleman HM, Kingshott RN, Wraith PK, et al. Randomized placebocontrolled crossover trial of CPAP for mild sleep apnea/hypopnea syndrome. Am J Respir Crit Care Med 1999;159:461-7.

27 Barnes M, Houston D, Worsnop CJ, et al. A randomized controlled trial of continuous positive airway pressure in mild obstructive sleep apnea. Am J Respir Crit Care Med 2002; 165:773-80.

28 Monasterio C, Vidal S, Duran J, et al. Effectiveness of continuous positive airway pressure in mild sleep apnea-hypopnea syndrome. Am J Respir Crit Care Med 2001; 164:939-43.

29 Hack M, Davies RJO, Mullins R, et al. Randomised prospective parallel trial of therapeutic versus subtherapeutic nasal continuous positive airway pressure on simulated steering performance in patients with obstructive sleep apnoea. Thorax 2000;55:224-31.

30 Redline S, Strauss ME, Adams N, et al. Neuropsychological function in mild sleep-disordered breathing. Sleep 1997;20:160-7.

31 Kim HC, Young T, Matthews CG, et al. Sleep-disordered breathing and neuropsychological deficits; a population-based study. Am J Respir Crit Care Med 1997; 156:1813-9.
32 Greenberg GD, Watson RK, Deptula D. Neuropsychological dysfunction in sleep apnea. Sleep 1987;10:254-62.

33 Naëgelé B, Thouvard V, Pépin J-L, et al. Deficits of cognitive executive functions in patients with sleep apnea syndrome. Sleep 1995;18:43-52.

34 Martin SE, Engleman HM, Deary IJ, et al. The effect of sleep fragmentation on daytime function. Am J Respir Crit Care Med 1996; 153:1328-32.

35 Kales A, Caldwell AB, Cadieux RJ, et al. Severe obstructive sleep apnea-ll: Associated psychopathology and psychosocial consequences. J Chron Dis 1985;38:427-34.

36 Ulfberg J, Carter N, Talback M, et al. Excessive daytime sleepiness at work and subjective work performance in the general population and among heavy snorers and patients with obstructive sleep apnea. Chest 1996;110:659-63.

37 Grunstein RR, Stenlöf JA, Hedner JA, et al. Impact of self-reported breathing disturbances on psychosocial performance in the Swedish obese subjects (SOS) study. Sleep 1995; 18:635-43.

38 Platon MJR, Sierra JE. Changes in psychopathological symptoms in sleep apnea patients after treatment with nasal continuous positive airway pressure. Int J Neurosci 1992;62:173-95.

39 Millman RP, Fogel BS, McNamara ME, et al. Depression as a manifestation of obstructive sleep apnea: reversal with nasal continuous positive airway pressure. J Clin Psychiatry 1989;50:348-51.

40 Fornas C, Ballester E, Arteta $E$, et al. Measurement of general health status in obstructive sleep apnea hypopnea patients. Sleep 1995;18:876-9.

41 Smith IE, Shneerson JM. Is the SF-36 sensitive to sleep disruption? A study in subjects with sleep apnea. J Sleep Res 1995;4:183-8.

42 Jenkinson C, Stradling J, Petersen S. Comparison of three measures of quality of life outcome in the evaluation of continuous positive airways pressure therapy for sleep apnoea. J Sleep Res 1997;6:199-204.

43 Flemons WW, Reimer MA. Development of a disease-specific health-related quality of life questionnaire for sleep apnea. Am J Respir Crit Care Med 1998; 158:494-503

44 Weaver TE, Laizner AM, Evans LK, et al. An instrument to measure functional status outcomes for disorders of excessive sleepiness. Sleep, 1997;20, 835-43.

45 Cohen J. Statistical power analysis for the behavioral sciences, 2nd ed. New Jersey: Erlbaum, 1988.

46 Engleman HM, Kingshott RN, Martin SE, et al. Cognitive function in the sleep apnoea/ hypopnoea syndrome (SAHS). Sleep 2000;23(Suppl 4):S102-8 